\title{
November Updates: People and Places
}

\section{Immunology awarded}

Eva Kaufmann has received the German Society of Immunology's first Werner-Muller Prize, an award given in recognition to young postdocs in recognitions of achievements in immunology. Kaufmann is recognized for her work with tuberculosis and the Bacillus Calmette-Guerin vaccine. In mouse models, she demonstrated a new mechanism for protection against the disease.

Kaufmann studied veterinary medicine and complete her doctorate degrees at Justus-Liebig-University Giessen in Germany. She is currently a postdoc at McGill University in Montreal, Canada.

Antibody biotech company Trianni sponsors the award, which is named for Werner Muller, a developer of transgenic and humanized antibody-producing mice.

\section{Lifetime Achievement}

Barry Rouse has received the 2018 Lifetime Excellence in Research Award from the American Veterinary Medical Association (AVMA). Rouse graduated with a veterinary degree from the University of Bristol in the UK and received a doctorate in immunology from the University of Guelph in Ontario, Canada.

The AVMA recognizes Rouse for his contributions to viral immunology and immunopathology. He currently works at the University of Tennessee College of Veterinary Medicine, studying the herpes simplex virus in mouse models.

\section{AAALAC awards UK Fellowship \\ AAALAC International has named Allan Thornhill as the 2018 UK recipient of its annual Fellowship Award. Thornhill is the head of biological services at the Institute of Cancer Research: Royal Cancer Hospital in London, where he manages the animal facility and Cancer Imaging Centre. The UK Fellowship is awarded to an IAT Registered (RAnTech) individual in recognition of their contributions to laboratory animal care and use.}

\section{Penn to map mouse models} Researchers at Pennsylvania State University have received a five year,

\section{Careers update}

Veterinarian John "Iain" Glen has been awarded the 2018 Lasker-DeBakey Clinical Medical Research Award for his contributions to the development the anesthesia propofol. Glen, a veterinary anesthesiologist, studied veterinary medicine at the University of Glasgow in Scotland. In 1972, he joined ICI Pharmaceuticals (later acquired by AstraZeneca), where he and his colleagues worked with lab mice to discover and develop a replacement for the anesthesia thiopentone. Propofol proved just as effective, but without the groggy after affects typical of thiopentone. It was approved for use in the United Kingdom and the United States in the late $80 \mathrm{~s}$. Glen is only the second veterinarian to receive a Lasker Award.

Animal research has featured in the work of this year's other recipients as well. The winners of the 2018 Albert Lasker Basic Medical Research Award, Michael Grunstein from the University of California, Los Angeles and C. David Allis from the Rockefeller University in New York, studied histones in nematode worms and mice (as well as yeast). Joan Argetsinger Steitz, a researcher from Yale, received the 2018 Lasker-Koshland Award for Special Achievement in Medical Science. Her work on RNA biology was conducted in rodent and other mammalian cells.

Each will receive a $\$ 250,000$ prize from the Albert and Mary Lasker Foundation.
$\$ 3$ million grant from the National Institute of Health Blueprint for Neuroscience Research to create high resolution, $3 \mathrm{~d}$ maps of blood vessels in the brains of young and old mice. Principal investigator Yongsoo Kim, an assistant professor of neural and behavioral sciences at the PSU College of Medicine, will collaborate with pathologist Keith Cheng and neural engineer Patrick Drew on the effort, the results of which will eventually be hosted online.

\section{Primates for reproductive health} Researchers at the Oregon National Primate Research Center (ONPRC) at Oregon Health and Science University in Portland, Oregon have received \$7 million from the National Institutes of Health to study polycystic ovary syndrome (PCOS). Led by Richard Stouffer, they will use female nonhuman primates to evaluate the impacts of high-fat diets and elevated testosterone levels on the animals' reproductive system. Along with the animal work, the grant will support a clinical trial of women with PCOS at the ONPRC's National Center for Translational Research in Reproduction and Infertility, to be directed by University of California Los Angeles Medical School professor Dan Dumesic.

\section{CAAT calls for refinement}

The Johns Hopkins Center for Alternatives to Animal Testing (CAAT) is currently accepting proposals for its 2019 ScienceBased Refinement Awards. The $\$ 5000$ awards will support projects to enhance housing, handling, and/or experimental procedures or to reduce animal use. Interested applicants can find more information at the CAAT website, www.caat.jhsph.edu. Applications are due by December $31^{\text {st }}, 2018$ and will be awarded and funded by April 1, 2019.

Published online: 23 October 2018 https://doi.org/10.1038/s41684-018-0175-8 